If the presence of $\mathrm{CP}$ or $\mathrm{CAC}$ score $>28$ were considered as subclinical atheromatosis disease (SAD), a total of 26 patients $(60.5 \%)$ were diagnosed. We performed multivariate regression analysis, CIMTm, CIMTmax, low High Density Lipid (HDL), high erythrocyte sedimentation rate (ESR) and age were independent factors for the presence of SAD.

Conclusions: SSc patients often have SAD which is misdiagnosed by CV risk charts. Plaque detection by carotid ultrasonography and CT CACscore are useful to detect SAD. The optimal cut-off point of CACscore in this study is 28. SAD detection would be indicated in elderly, patients with low HDL or high ESR. The measure of CIMT could be useful in some cases.

Disclosure of Interest: None declared

DOI: 10.1136/annrheumdis-2017-eular.1515

\section{SAT0341 PILOT STUDY: HOME MANAGEMENT OF ILOPROST IN THE MICROPUMP/24 HOURS, IN PATIENTS SUFFERING FROM SCLERODERMA, FOLLOWED FOR 12 MONTHS}

G. Pistone, R. Daita, C. Arcuri, N. Catalano, S. Liuzza, C. Rinollo, G. D’Aguanno, A. Allotta, M. Lo Monaco, R. Mallaci Bocchio, G. Natoli, S. Corrao. AZ.OSP.ARNAS Civico, PALERMO, Italy

Background: evaluate the effectiveness on Raynaud's phenomenon and on digital ulcers, safety and side effects, during intravenous infusion of iloprost therapy in patients with diffuse and limited scleroderma

Objectives: the effectiveness of the therapy was assessed at 0 and 12 months, on the basis of daily number of episodes of Raynaud's phenomenon, evaluation of digital ulcers changes of the vascular nail bed to capillaroscopio, evaluation of pulmonary hypertension.

Security according to the appearance of adverse events

Methods: They were enrolled consecutively, for a period of 12 months, 12 patients, 9 women and 2 men, 9 suffering from diffuse scleroderma (positive Scl70) and 3 suffering from limited scleroderma (positive anticentromere), middle age 52,91 years, mean age of disease 7,8 years, 11 non-smoking. They were subjected to infusion of iloprost $1 / 2$ vial diluted in $25 \mathrm{cc}$ of saline solution to 0,6 $\mathrm{ml} / \mathrm{h}$, in micropump Infonde for 24 consecutive hours, for 4 days a month for 12 months, 10 patients with peripheral lines and 2 with central venous access.

Results: of 12 patients with the Raynaud's phenomenon, there was a reduction in the daily number in 4 patients, unchanged in 8 patients. Digital ulcers disappeared in 6 patients, they were unchanged in 5 patients, improved although present in 1 patient. In 7 patients capillaroscopic framework remained unchanged, in 5 patients improved capillary density. 10 patients had no pulmonary hypertension, 2 patients had pulmonary hypertension, no change in the two groups at T0 and T 12. None of the patients experienced side effects during the infusion.

Conclusions: the cyclical infusion therapy iloprost prevented the onset of ulcers in patients at TO not presented or the appearance of new ulcers; it has contributed to the reduction in the daily number of Raynaud's phenomenon and also allowed to reach the same effective dose of a vial of iloprost to dilute in $50 \mathrm{cc}$ of saline solution, allowing the patient to perform at home therapy with greater compliance. In terms of safety none of the patients experienced side effects or adverse effects Disclosure of Interest: None declared

DOI: 10.1136/annrheumdis-2017-eular.5990

\section{SAT0342 TRANSTHORACIC ECHOCARDIOGRAPHY TO QUANTIFY PULMONARY VASCULAR RESISTANCE IN PATIENTS WITH SYSTEMIC SCLEROSIS}

S. Billet ${ }^{1}$, T. Chollet ${ }^{1}$, P. Fournier ${ }^{1}$, L. Tetu ${ }^{2}$, G. Prévot ${ }^{2}$, M. Galinier ${ }^{1}$, D. Carrié ${ }^{1}$, O. Lairez ${ }^{1}$, G. Pugnet ${ }^{3}{ }^{1}{ }^{1}$ Service de Cardiologie, CHU Rangueil; ${ }^{2}$ Service de Pneumologie, CHU Larrey; ${ }^{3}$ Service de Médecine Interne, CHU Toulouse Purpan, Toulouse, France

Background: One of the major causes of systemic sclerosis (SSc)-related death is pulmonary arterial hypertension, which develops in $12-15 \%$ of patients with SSc and accounts for $30-40 \%$ of deaths. Consequently, monitoring of pulmonary arterial pressure (PAP) and resistances is essential in patients with SSc. Abbas formula performed by transthoracic echocardiography (TTE) was reported as a good tool to quantify pulmonary vascular resistances (PVR).

Objectives: Explore the accuracy of TTE and Abbas formula to quantify PVR in patients with SSc.

Methods: All consecutive patients with SSc, diagnosed according to the 2013 ACR/EULAR criteria, or the LeRoy and Medsger criteria for diffuse or limited subsets classification, had within $24 \mathrm{H}$ a Doppler echocardiographic examination and right-heart catheterization were performed. The ratio of peak tricuspid regurgitant velocity (TRV, $m s$ ) to the right ventricular outflow tract time-velocity integral $\left(\mathrm{TVI}_{\mathrm{RVOT}}, \mathrm{cm}\right.$ ) obtained by Doppler echocardiography (TRV/TVI RVOT $_{\text {) }}$ ) was then correlated with invasive PVR measurements using regression analysis. An equation was modeled to calculate PVR in Wood units (WU) using echocardiography, and the results were compared with invasive PVR measurements [1].

Results: Thirty-three consecutive patients were included, 13 (39.4\%) were male and the mean age was $64.6 \pm 12.1$ years. Most were classified as limited cutaneous SSc (IcSSc; $\mathrm{n}=29,87.9 \%$ ). All patients tested positive for antinuclear antibodies, $18(21.2 \%)$ for anti-scleroderma-70, 7 (54.5\%) for anticentromere antibodies and
$2(6.1 \%)$ for anti-RNA polymerase III antibodies. Mean and systolic PAP were $31 \pm 9$ and $53 \pm 16 \mathrm{mmHg}$ respectively. There was a good correlation between right ventricle to right atrium gradient pressure assessed by TTE and $\mathrm{RHC}(\mathrm{R}=0.620$, $\mathrm{P}<0.001)$. RVP assessed by Abbas formula $(2.6 \pm 1.0$ wood units) were well correlated with RVP assessed by RHC (4.8 $2.3 ; \mathrm{R}=0.446, \mathrm{P}=0.013)$.

Figure. Correlations between right ventricular to right atrium gradient and pulmonary arterial resistances assessed by transthoracic echocardiography and right heart catheterization
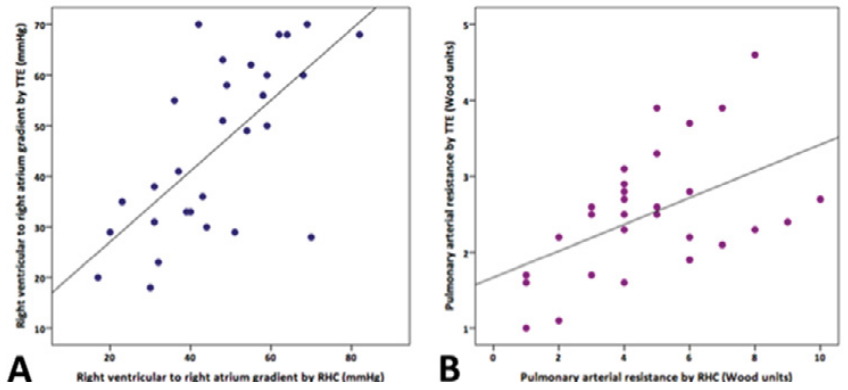

Conclusions: Doppler echocardiography using Abbas formula may provide a reliable, noninvasive method to determine PVR in SSc patients.

\section{References:}

[1] Abbas AE, Fortuin FD, Schiller NB, Appleton CP, Moreno CA, Lester SJ. A simple method for noninvasive estimation of pulmonary vascular resistance. $J$ Am Coll Cardiol. 2003 Mar 19;41(6):1021-7.

Disclosure of Interest: None declared

DOI: 10.1136/annrheumdis-2017-eular.2081

\section{SAT0343 SCREENING OF PULMONARY ARTERIAL HYPERTENSION IN PATIENTS WITH SYSTEMIC SCLEROSIS USING DETECT ALGORITHM - VALIDATION IN THE COHORT OF JAPANESE SINGLE CENTER}

H. Yasuoka ${ }^{1}$, Y. Shirai ${ }^{2}$, Y. Tamura ${ }^{3}$, T. Takeuchi ${ }^{1}$, M. Kuwana ${ }^{2} .{ }^{1}$ Division of Rheumatology, Department of Internal Medicine, Keio University School of Medicine; ${ }^{2}$ Departmenrt of Allergy and Rheumatology, Nippon Medical School Graduate School of Medicine; ${ }^{3}$ International University of Health and Welfare Mita Hospital, Tokyo, Japan

Background: Pulmonary arterial hypertension (PAH) complicated with systemic sclerosis (SSc) has the worst prognosis in $\mathrm{PAH}$ associated with other connective tissue diseases (1) and is one of leading cause of death in patients with SSc (2). To improve prognosis in SSc patients, earlier detection and diagnosis of $\mathrm{PAH}$ by annual screening is recommended even in asymptomatic patients (3). To effectively detect PAH in patients with SSc at earlier phase, DETECT algorithm is reported as a good tool to identify candidates who need right heart catheterization $(\mathrm{RHC})$, with high sensitivity (4). However, its usefulness has not been validated in Japanese cohorts.

Objectives: To validate the effectiveness of DETECT algorithm in the Japanese single center cohort.

Methods: Patients with SSc who visited Keio University Hospital between 2005 and 2016 were included in the study. Patients over 18 years old, disease duration more than 3 years, and DLCO predicted less than $60 \%$ were selected and clinical information was retrospectively collected from records. The sensitivity, specificity, and negative and positive predictive values of the algorithm based on the result of $\mathrm{RHC}$ evaluation were calculated in a cohort of $\mathrm{PAH}$ patients and non-PAH patients, in whom $\mathrm{RHC}$ data were available. Validation with patients with data minimally-required for algorithm were also examined.

Results: Three hundred four cases were visited our hospital during from 2005 to 2016. Patients who fulfilled criteria and had data minimally-required for algorithm were 126 cases. Of 126 patients, 50 were examined $\mathrm{RHC}$ evaluation and patients diagnosed as pulmonary hypertension were $26(21 \%)$ and $21(15 \%)$ were $\mathrm{PAH}$. When a cohort of $\mathrm{PAH}$ patients and non-PAH patients with $\mathrm{RHC}$ data was applied to DETECT algorithm, referral rate to $\mathrm{RHC}$ evaluation was $78 \%$, missed diagnosis of $\mathrm{PAH}$ was $0 \%$. Sensitivity/specificity for detecting PAH patients were $100 \% / 42 \%$, and positive/negative predictive values were $60 \% / 100 \%$, respectively. Evaluation of 126 patients with data minimally-required for algorithm was also examined. Referral rate to $\mathrm{RHC}$ evaluation was $43 \%$, missed diagnosis of $\mathrm{PAH}$ was $0 \%$, and sensitivity/specificity and positive/negative predictive values for detecting $\mathrm{PAH}$ patients were $100 \% / 72 \%, 43 \% / 100 \%$, respectively. However, there was a patient who was initially excluded at Step 1, but developed PAH one year later.

Conclusions: The DETECT algorithm was reassured as a good tool to effectively screening tool for $\mathrm{PAH}$ in SSc patients. However, we have to keep in mind that unnecessity of $\mathrm{RHC}$ referral judged by this algorithm does not guarantee the patient to be free from future development of PAH.

References:

[1] Condliffe R et al. Am J Respir Crit Care Med 179:151-157, 2009.

[2] Steen VD et al. Ann Rheum Dis 66:940-944, 2007 
[3] Galiè $\mathrm{N}$ et al. Eur Heart J 37:67-119, 2016.

[4] Coghlan JG et al. Ann Rheum Dis 73:1340-1349, 2014.

Disclosure of Interest: None declared

DOI: 10.1136/annrheumdis-2017-eular.4608

\section{SAT0344 DISEASE RELATED MALNUTRITION IN SYSTEMIC SCLEROSIS AND ASSOCIATED FACTORS: A CROSS-SECTIONAL STUDY}

I. Türk ${ }^{1}$, N. Cuzdan Coskun ${ }^{2}$, V. Ciftci ${ }^{3}$, D. Arslan Taş ${ }^{1}$, M.C. Doğan ${ }^{4}$, I. Unal ${ }^{5}$ ${ }^{1}$ Department of Internal Medicine, Division of Rheumatology; ${ }^{2}$ Department of Physical Medicine and Rehabilitation, Division of Rheumatology; ${ }^{3}$ Faculty of Dentistry, Department of Pediatric Dentistry; ${ }^{4}$ Department of Pediatric Dentistry; ${ }^{5}$ Department of Biostatistics, Çukurova University, Adana, Turkey

Background: The risk of malnutrition increases in patients with systemic sclerosis (SSc) which has a negative prognostic effect. Additionally malnutrition is a significant cause of morbidity and mortality.

Objectives: The aim of this study was to evaluate the associations between malnutrition and clinical features of the disease, depression in SSc patients.

Methods: Concomitant SSc patients followed in our outpatient and inpatient clinics were enrolled in the study. Skin involvement was assessed with modified Rodnan skin score (mRSS), joint/tendon involvement with finger-tip to palm distance (FTP). Interstitial lung disease (ILD) and heart involvement were evaluated with clinical and radiological methods. Patients were questioned for dysphagia and gastroesofageal reflux as an indicator of esophageal involvement, early satiety and vomiting as gastric involvement, and diarrhea, constipation and bloating as bowel involvement. ${ }^{1}$ Interincisal distance measurement was used to assess the maximal mouth opening capacity. Malnutrition risk was assessed by the Malnutrition universal screening tool (MUST). The Beck Depression Inventory (BDI) was used for measuring the severity of depression. sWe examined associations between malnutrition risk and clinical features of the disease, depression in SSc patients. Results: Ninety eight SSc patients with 69 diffuse and 29 limited type of the disease were enrolled in the study. $84.7 \%$ of the patients were female and the mean age was $52.67 \pm 11.28$ years. According to MUST scores $61.2 \%$ of patients have low, $15.3 \%$ medium and $23.5 \%$ high risk for malnutrition. We found no difference between the malnutrition risk among genders $(p=0.065)$. mRSS and FTP were significantly different between malnutrition risk groups $(p=0.005,0.050$ respectively). Malnutrition risk was higher with patients with ILD than the patients without ILD $(p=0.044)$. Malnutrition risk was higher with patients with bowel involvement than the patients without bowel involvement. $(p=0.021)$.

Interincisal distance was significantly different between malnutrition risk groups $(p=0.003) .78 .7 \%$ of SSc patients have BDI scores $\geq 10,54.3 \%$ of SSc patients have $\mathrm{BDI}$ scores $\geq 17$. BDI scores were significantly different between malnutrition risk groups $(p<0.001)$. Factors affecting malnutrition risk were evaluated with logistic regression analysis. Interincisal distance and bowel involvement were found to be the most relevant factors for malnutrition risk. SSc patients with bowel involvement have 2.519-fold increased risk of malnutrition compared to patients without bowel involvement (95\% Cl 1.039-6.105). Every $1 \mathrm{~mm}$ decrement in interincisal distance was associated with 1.101 fold increase in malnutrititon risk of SSc patients $(95 \% \mathrm{Cl} 1.032-1.176)$.

Conclusions: Malnutrition is common in SSc patients. Malnutrition risk is associated with skin, tendon/joint involvement, DU count, ILD, bowel involvement, microstomia and depression severity.

\section{References:}

[1] Walker UA, Tyndall A, Czirják $L$ et al. Clinical risk assessment of organ manifestations in systemic sclerosis: a report from the EULAR Scleroderma Trials And Research group database. Ann Rheum Dis. 2007;66:754-63.

Disclosure of Interest: None declared

DOI: 10.1136/annrheumdis-2017-eular.6162

\section{SAT0345 THE IMPACT OF GASTROINTESTINAL INVOLVEMENT IN HEALTH-RELATED QUALITY OF LIFE IN PATIENTS WITH SYSTEMIC SCLEROSIS}

J. Caetano ${ }^{1}$, J. Branco ${ }^{2}$, S. Oliveira ${ }^{1}$, J. Delgado Alves ${ }^{1,3} \cdot{ }^{1}$ Systemic Autoimmune Diseases Unit, Department of Medicine IV; ${ }^{2}$ Department of Gastroenterology, Fernando Fonseca Hospital, Amadora; ${ }^{3}$ CEDOC, NOVA Medical School, Lisbon, Portugal

Background: Apart from skin involvement, the gastrointestinal (Gl) system is the second most commonly involved organ in systemic sclerosis (SSc), affecting over $80 \%$ of the patients. Although rarely being a direct cause of death, it is associated to a high morbidity and to a significant impairment in health-related quality of life (HRQoL). Few data are published considering the influence of GI manifestations in the quality of life of SSc patients.

Objectives: The purpose of this study is to characterize the impact of Gl involvement in HRQoL in SSc, and to compare it with that in patients with inflammatory bowel disease (IBD).

Methods: 29 consecutive SSc patients with GI involvement were selected from a cohort of 44 SSc patients, followed in a single referral centre and classified according to 2013 ACR/EULAR criteria. Comparative analysis was made with 24 consecutive patients with IBD without arthritis (14 Crohn's disease; 10 - ulcerative colitis). Health Assessment Questionnaire (HAQ-DI) and Short Form 36 (SF36), physical component summary (PCS) and mental component summary (MCS) scales, were used to assess HRQoL in both groups. The UCLA Scleroderma Clinical Trial Consortium Gastrointestinal Tract 2.0 (UCLA SCTG GIT 2.0) was performed in SSc patients to assess the severity of $\mathrm{Gl}$ involvement. Clinical data were obtained by medical records review. T-test and Fisher's exact test were used to compare binary variables. Pearson's correlation was used for continuous variables.

Results: The most common Gl segments involved in SSc patients were the esophagus in $90 \%$, the stomach in $60 \%$ and the bowel in $48 \%$, while the anorectum was involved in a smaller percentage (10.3\%). The mean UCLA SCTG GIT 2.0 score was $0.64 \pm 0.51$, compatible with a moderate severity, with higher scores obtained for reflux, distention and emotional wellbeing. The mean HAQ-DI score was 1.13 \pm 0.57 (0-best health), and the mean PCS and MCS scores were $35.2 \pm 9.4$ and $35.3 \pm 11.4$ (100-best health), respectively. These scores were significantly worse than in SSc patients without GI involvement (HAQ-DI $p=0.001$; PCS $-p=0.04$ and MCS $-p=0.005$ ). There was a significant correlation between higher UCLA SCTG GIT 2.0 scores and worse quality of life evaluated by HAQ-DI $(r=0.42, p=0.03)$, but the same correlation was not found for the SF36 components. Comparing with IBD patients, SSc patients with Gl involvement had worse life quality, with statistical significantly higher HAQ-DI $(p<0.001)$ and lower PCS $(p<0.001)$ and MCS $(p=0.01)$ scores.

Conclusions: Gl involvement in SSc significantly impaired patient's quality of life. The impact of Gl involvement in HRQoL of SSc patients was more severe than in IBD patients. Although physical components were relevant, with esophagus being most frequently involved, mental components associated with Gl involvement significantly compromised HRQoL in SSc. Therefore, the assessment of SSc patients using clinical severity measure tools, similar to UCLA SCTG GIT 2.0 score, is crucial for a better characterization of the disease and to an optimized clinical approach.

Disclosure of Interest: None declared

DOI: 10.1136/annrheumdis-2017-eular.2496

\section{SAT0346 SYSTEMIC SCLEROSIS'S EARLY STAGES AND ITS SIGNIFICANCE IN DAILY PRACTICE}

J.P. Abreu, I. Almeida, A. Marinho, C. Vasconcelos. Unidade de Imunologia Clínica, Centro Hospitalar do Porto, Porto, Portugal

Background: Systemic Sclerosis (SSc) is an autoimmune disease, characterized by skin and internal organs fibrosis. Its clinical manifestations are heterogeneous, hence, the definitive diagnosis of SSc rely on scores, such as American College of Rheumatology (ACR) for SSc. Leroy and Medsger, proposed the potential criteria for the classification of early stages of SSc, due to the insufficient sensitivity previous methods. Lately EUSTAR proposed the concept of Very Early SSc, defined by the presence of 3 red flag - RF, Puffy Fingers and ANA; if 2 of these are present, capilaroscopy and specific antibody must be accessed; if one of the last positive, further search for Systemic involvement must be taken in account. Objectives: We propose to: analyse a group of patients that had been diagnosed with early stages of SSc; study the presence of systemic involvement (at the time of diagnosis and throughout the follow-up), and the possible predictors for progression to definitive SSc.

Methods: The patients were selected by consulting the clinical data from patients followed in our hospital with diagnose of early stages of SSc. Very Early SSc criteria were used to define early stage SSc. The data concerning red flags, capilaroscopy, specific auto-antibodies, systemic involvement (Gastrointestinal, Pulmonary, Cardiac, Kidney, Muscoloskeletal).

Results: We obtained a sample of 70 patients, 65 being female subjects $(92,9 \%)$, with mean age of diagnosis 47.9 years (SD 13,6). 16 patients $(22,9 \%)$ were classified as Pre-scleroderma at the admission, 16 as Early SSc (22,9\%), 15 $(21,4 \%)$ as Very Early SSc, $16(22,9 \%)$ as possible SSc, $6(8,6 \%)$ as Limited SSc (despite not scoring to ACR criteria) and $1(1,4 \%)$ as Sin Scleroderma SSc. 63 patients $(90 \%)$ had RP, $15(21,4 \%)$ had puffy fingers, $63(90 \%)$ ANA positivity 11 patients $(15,7 \%)$ had 1 red flag, $45(64,3 \%)$ had 2 red flags and $13(18,6 \%)$ had 3 red flags.

54 patients $(78.3 \%)$ were positive to specific SSc autoantibodies; 47 patients $(71.2 \%)$ had capiloroscopy compatible with SSc in different stages $(17.1 \%$ Early, $12,9 \%$ Active, $4,3 \%$ Late and $32,9 \%$ compatible). 54 patients $(77,1 \%)$ scored for Very Early SSc.

At the moment of the diagnosis, 56 patient $(81,2 \%)$ had systemic involvement. 34 patients $(48,6 \%)$ scored to the definitive diagnosis by ACR 2013 criteria, after 2,56 years in mean (SD 2.51) of first diagnosis.

No correlation was found in what concerns to predictors of progression to SSc or systemic involvement.

Conclusions: This study shed a light on the importance of the recognition of SSc's early stages, since a significant part of these patients have systemic involvement at the moment of diagnosis, yet not scoring to ACR 2013 criteria. Almost half of the patients scored to definite diagnosis of SSc at the moment of the last appointment. The importance of these findings rely on the possibility to identify systemic involvement and address it in time to prevent/delay further disease progression.

References:

[1] Avouac J, Matucci-Cerinic M, Miniati I, Denton CP. Systemic Sclerosis. EULAR Online Course on Rheumatic Disease 2016 